\title{
The Main Eigenvalues of the Seidel Matrix
}

\author{
HOUQING ZHOU*
}

\begin{abstract}
Let $G$ be a simple graph with vertex set $V(G)$ and $(0,1)$ adjacency matrix $A$. As usual, $A^{*}(G)=J-I-2 A$ denotes the Seidel matrix of the graph $G$. The eigenvalue $\lambda$ of $A$ is said to be a main eigenvalue of $G$ if the eigenspace $\varepsilon(\lambda)$ is not orthogonal to the all-1 vector e. In this paper, relations between the main eigenvalues and associated eigenvectors of adjacency matrix and Seidel matrix of a graph are investigated.
\end{abstract}

\section{INTRODUCTION}

Let $G$ be a simple graph with $n$ vertices. We write $V(G)$ for the vertex set of $G$, and $E(G)$ for the edge set of $G$. The spectrum of the graph $G$ consists of the eigenvalues $\lambda_{1} \geq \lambda_{2} \geq \cdots \geq \lambda_{n}$ of its $(0,1)$ adjacency matrix $A=A(G)$ and is denoted by $\sigma(G)$. The Seidel spectrum of $G$ consists of the eigenvalues $\lambda_{1}^{*} \geq \lambda_{2}^{*} \geq \cdots \geq \lambda_{n}^{*}$ of its $(0,-1,1)$ adjacency matrix $A^{*}=A^{*}(G)$ and its denoted by $\sigma^{*}(G)$. Let $P_{G}(\lambda)=|\lambda I-A|$ and $P_{G}^{*}(\lambda)=\left|\lambda I-A^{*}\right|$ denote the characteristic polynomial and the Seidel characteristic polynomial, respectively.

For a real symmetric matrix $A$, an eigenvalue of $A$ is called simple if its algebraic multiplicity is one, and the eigenvalue $\lambda$ of $A$ is said to be a main eigenvalue of $G$ if the eigenspace $\varepsilon(\lambda)$ is not orthogonal to the all-1 vector e. Any real symmetric matrix $A$ has at least one main eigenvalue. Furthermore, matrix $A$ has exactly one main eigenvalue if and only if the vector $\mathbf{e}=(1,1, \ldots, 1)^{T}$ is an eigenvector of $A$. For a graph $G$, its main eigenvalues are those of $A(G)$, and $G$ has exactly one main eigenvalue if and only if $G$ is a regular graph. There are many results and their applications on the main eigenvalues of graphs, see[1],[2],[3],[4],[6],[7], but it is still an open problem to characterize the graphs with exactly $l(l \geq 3)$ main eigenvalues(as the case $l=2$ has been settled, see [4],[5]). It is well known that if the graph

2000 Mathematics Subject Classification. Primary 05C50, 05C35.

Key words and phrases. Graph spectra, Main eigenvalues, Seidel matrix.

${ }^{*}$ The author was supported in part by Scientific Research Fund of Hunan Provincial Education Department \#06C755; and the Hunan Provincial Natural Science Foundation of China \#2008FJ3090. 
$G$ is $r$-regular graph, in other words, $G$ has exactly one main eigenvalue, then( [1] p. 30)

$$
P_{G}^{*}(\lambda)=(-1)^{n} 2^{n} \frac{\lambda+1+2 r-n}{\lambda+1+2 r} P_{G}\left(-\frac{\lambda+1}{2}\right) .
$$

Hence the Seidel spectrum of regular graph is determined by its adjacency spectrum.

The aim of this paper is to prove that the main Seidel eigenvalues of a graph are recoverable by the main eigenvalues of adjacency matrix and associated eigenvectors and give a method for computing the main Seidel eigenvalues in terms of the main eigenvalues and associated eigenvectors of adjacency matrix.

The rest of the paper is organized as follows. In Section 2 contains some definitions. In Section 3 we will describe the relation between main eigenvalues of $G$ and main eigenvalues of $A^{*}(G)$, and prove several theorems on the main eigenvalue of graphs.

\section{Some BASIC NOTIONS}

Let $A$ have spectral decomposition

$$
A=\mu_{1} P_{1}+\mu_{2} P_{2}+\cdots+\mu_{m} P_{m} .
$$

The main angles of $G$ are the numbers $\beta_{1}, \beta_{2}, \ldots, \beta_{m}$, where $\beta_{i}=\frac{1}{\sqrt{n}}\left\|P_{i} \mathbf{e}\right\|$ $(i=1,2, \ldots, m)$. These are the cosines of the angles between $\mathbf{e}$ and the eigenspaces of $A$, and so $\mu_{i}$ is a main eigenvalue if and only if $\beta_{i} \neq 0$. Since $\|\mathbf{e}\|^{2}=\sum_{i=1}^{m}\left\|P_{i} \mathbf{e}\right\|^{2}$, we have $\sum_{i=1}^{m} \beta_{i}^{2}=1$. The main eigenvalues include the index (largest eigenvalue) of $G$ because there exists a corresponding eigenvector with no negative entries, see [1].

We take the main eigenvalues of $G$ to be $\mu_{1}, \mu_{2}, \ldots, \mu_{s}$, with $\mu_{1}$ the index of $G$; no further ordering is assumed for $\mu_{2}, \mu_{3}, \ldots, \mu_{s}$.

First, we introduce some notation and preliminaries which will be useful to obtain the main results.

It is not difficult to see the following lemmas:

Lemma 2.1. (see [1]) The relation between the characteristic polynomial $P_{G}(\lambda)$ of a graph $G$ and the characteristic polynomial $P_{G}^{*}(\lambda)$ of the Seidel adjacency matrix $A^{*}(G)$ of $G$ can be written in the form

$$
P_{G}(\lambda)=\frac{(-1)^{n}}{2^{n}} \cdot \frac{P_{G}^{*}(-2 \lambda-1)}{1+\frac{1}{2 \lambda} H_{G}\left(\frac{1}{\lambda}\right)} .
$$

Lemma 2.2. (see [9]) If $N_{k}$ denotes the number of walks of length $k$ in $G$, then

$$
N_{k}=n \sum_{i=1}^{s} \mu_{i}^{k} \beta_{i}^{2} .
$$


According to [1], the walk generating function $H_{G}(t)$ is defined by $H_{G}(t)=\sum_{k=0}^{+\infty} N_{k} t^{k}$, and it follows from Lemma 2.2 that

$$
H_{G}(t)=\sum_{i=1}^{s} \frac{n \beta_{i}^{2}}{1-\mu_{i} t} .
$$

Using above lemma, we see that the main eigenvalues of $A^{*}(G)$ are determined by the main eigenvalues of $G$.

\section{MAIN RESULTS}

We proceed now to the investigation of the main Seidel eigenvalues of a graph $G$. We shall apply above lemma and a result from [1], the following result is immediately obtained.

\section{Theorem 3.1.}

$$
P_{G}^{*}(\lambda)=(-2)^{n} P_{G}\left(-\frac{\lambda+1}{2}\right)\left(1-n \sum_{i=1}^{s} \frac{\beta_{i}^{2}}{\lambda+1+2 \mu_{i}}\right) .
$$

Proof. According to (2.2) and (2.3), by a straightforward calculation, hence we have (3.1).

Note that $A^{*}(G)=J-I-2 A(G)$, where the symbol $J$ denotes a square matrix all of whose entries are equal to $1, I$ means a unit matrix in general, respectively. If $\alpha$ is an eigenvector of $A(G)$ with eigenvalue $\mu$ such that $\mathbf{e}^{T} \alpha=0$, then $\alpha$ is also an eigenvector of $A^{*}(G)$ with eigenvalue $-1-2 \mu$, since $A^{*}(G) \alpha=(J-I-2 A(G)) \alpha=J \alpha-\alpha-2 A(G) \alpha=(-1-2 \mu) \alpha$. In other words, the non-main eigenvalues of $A^{*}(G)$ are determined by those of $A(G)$. Using this fact, we can simplify Equation (3.1) so that it involves only the main eigenvalues $\mu_{1}, \mu_{2}, \ldots, \mu_{s}$ and $\lambda_{1}^{*}, \lambda_{2}^{*}, \ldots, \lambda_{s}^{*}$ of $A(G)$ and $A^{*}(G)$, respectively, i.e.

$$
\prod_{i=1}^{s}\left(\lambda-\lambda_{i}^{*}\right)=\prod_{i=1}^{s}\left(\lambda+1+2 \mu_{i}\right)\left(1-n \sum_{i=1}^{s} \frac{\beta_{i}^{2}}{\lambda+1+2 \mu_{i}}\right) .
$$

Using Equation (3.2) for both $A(G)$ and $A^{*}(G)$, we can see the main eigenvalues of $A^{*}(G)$ are determined by the main eigenvalues and corresponding eigenvector of $A(G)$. But we can say more.

Theorem 3.2. Suppose that $\mu_{k}$ is a main eigenvalue of $A(G)$, then $-1-2 \mu_{k}$ cannot be a main eigenvalue of $A^{*}(G)$.

Proof. By evaluating Equation (3.1) at $-1-2 \mu_{k}$, we have

$$
\prod_{i=1}^{s}\left(-1-2 \mu_{k}-\lambda_{i}^{*}\right)=2^{s} \prod_{i=1, i \neq k}^{s}\left(\mu_{i}-\mu_{k}\right)\left(1-n \sum_{i=1}^{s} \frac{\beta_{i}^{2}}{2\left(\mu_{i}-\mu_{k}\right)}\right) .
$$

Hence for $i=1,2, \ldots, s, \lambda_{i}^{*} \neq-1-2 \mu_{k}$. 
For example, if $G$ is the cycle $C_{4}$ then its the main eigenvalue is 2, via calculation, show that -5 is non-main eigenvalue of $A^{*}(G)$, its main eigenvalue is 3 .

A consequence of this theorem is the following.

Corollary 3.3. Suppose that $\mu$ is a simple main eigenvalue of $A(G)$. Then $-1-2 \mu \notin \sigma^{*}(G)$.

Now, we give the following lemma.

Lemma 3.4. Let $\mu \in \sigma(G)$. Then $-1-2 \mu \in \sigma^{*}(G)$ if and only if $\mathbf{e}^{T} \alpha=0$ for some eigenvector $\alpha$ corresponding to the eigenvalue $\mu$ of $A(G)$.

Proof. Sufficiency follows from the Theorem 3.1.

To prove necessity. Assume that $-1-2 \mu \in \sigma^{*}(G)$ and note that $\mu$ cannot be simple main eigenvalue of $A(G)$ by Corollary 3.3. By Theorem 3.2, $-1-2 \mu$ is not a main eigenvalue of $A^{*}(G)$. Thus $A^{*}(G)$ has an eigenvector $\alpha$ corresponding to $-1-2 \mu$ such that $\mathbf{e}^{T} \alpha=0$ and $\alpha$ is also an eigenvector of $A(G)$ corresponding to $\mu$.

Next, we present the main result of this note that the main eigenvalues and associated eigenvectors of $A^{*}(G)$ are recoverable from those of $A(G)$.

Theorem 3.5. Let $\mu_{1}, \mu_{2}, \ldots, \mu_{s}$ be the main eigenvalues of the graph $G$, and let $\alpha_{1}, \alpha_{2}, \ldots, \alpha_{s}$ be corresponding orthonormal eigenvectors. Let $E$ be the $s \times s$ matrix whose (i,j)-entry is $\mathbf{e}^{T} \alpha_{i} \mathbf{e}^{T} \alpha_{j}$, and let $M=E-I-$ $2 \operatorname{diag}\left(\mu_{1}, \mu_{2}, \ldots, \mu_{s}\right)$. Then eigenvalues of $M$ are precisely the main eigenvalues of $A^{*}(G)$. Moreover, if $c=\left(c_{1}, c_{2}, \ldots, c_{s}\right)^{T}$ is an eigenvector of $M$ corresponding to the eigenvalue $\lambda^{*}$, then $\sum_{i=1}^{s} c_{i} \alpha_{i}$ is an eigenvector of $A^{*}(G)$ corresponding to $\lambda^{*}$.

Proof. Let $\lambda^{*}$ be a main eigenvalue of $A^{*}(G)$ with the corresponding eigenvector $\alpha^{*}$. Since any eigenvector $\alpha$ of $A(G)$ such that $\mathbf{e}^{T} \alpha=0$ is also an eigenvector of $A^{*}(G)$ and vice versa, two spaces spanned by the eigenvectors of $A(G)$ and $A^{*}(G)$ the sum of whose entries is zero are identical. Equivalently, the eigenvectors associated with the main eigenvalues of $A^{*}(G)$ span the same space as that of $A(G)$. Thus we can express $\alpha^{*}$ as a linear combination of eigenvectors $\alpha_{1}, \alpha_{2}, \ldots, \alpha_{s}, \alpha^{*}=\sum_{i=1}^{s} c_{i} \alpha_{i}$. Hence $A(G) \alpha^{*}=$ $\sum_{i=1}^{s} c_{i} \mu_{i} \alpha_{i}$. As $A(G)=\frac{1}{2}\left(J-I-A^{*}(G)\right)$, so $A(G) \alpha^{*}=\frac{1}{2}\left(J-\alpha^{*}-\lambda^{*} \alpha^{*}\right)$. Thus $J \alpha^{*}=2 A(G) \lambda^{*}+\left(1+\lambda^{*}\right) \alpha^{*}$. Combining above two expressions we get

$$
\left(\mathbf{e}^{T} \alpha^{*}\right) \mathbf{e}=\left(\mathbf{e}^{T} \mathbf{e}\right) \alpha^{*}=J \alpha^{*}=\sum_{i=1}^{s} c_{i}\left(2 \mu_{i}+1+\lambda^{*}\right) .
$$

Taking the scalar product of both side with $\alpha_{i}, i=1,2, \ldots, s$. We obtain

$$
\mathbf{e}^{T} \alpha^{*} \mathbf{e}^{*} \alpha_{j}=\sum_{i=1}^{s} c_{i} \mathbf{e}^{T} \alpha_{i} \mathbf{e}^{T} \alpha_{j}=\left(2 \mu_{j}+1+\lambda^{*}\right) c_{j} .
$$


In matrix form, the set of equations represented by (7) is

$$
\left(E-I-2 \operatorname{diag}\left(\mu_{1}, \mu_{2}, \ldots, \mu_{s}\right)\right) c=\lambda^{*} c .
$$

Thus $\lambda^{*}$ is an eigenvalue of $M$ with corresponding eigenvector $c$, and Theorem follows.

Similarly, the main eigenvalues and associated eigenvectors of $A(G)$ are recoverable from those of $A^{*}(G)$.

Theorem 3.6. Let $\lambda_{1}^{*}, \lambda_{2}^{*}, \ldots, \lambda_{l}^{*}$ be the main eigenvalues of $A^{*}(G)$ and $\alpha_{1}^{*}, \alpha_{2}^{*}, \ldots, \alpha_{l}^{*}$ be the associated orthonormal eigenvectors. Let $E$ be the $l \times l$ matrix whose $(i, j)$-entry is $\mathbf{e}^{T} \alpha_{i}^{*} \mathbf{e}^{T} \alpha_{j}^{*}$, and $M^{*}=\frac{1}{2}\left(E-I-\operatorname{diag}\left(\lambda_{1}^{*}, \lambda_{2}^{*}, \ldots, \lambda_{l}^{*}\right)\right)$. Then eigenvalues of $M^{*}$ are precisely the main eigenvalues of $A(G)$. Further more, if $b=\left(b_{1}^{*}, b_{2}^{*}, \ldots, b_{l}^{*}\right)^{T}$ is an eigenvector that corresponding to an eigenvalue $\mu^{*}$ of $M^{*}$, then $\sum_{j=1}^{l} b_{j}^{*} \alpha_{j}^{*}$ is an eigenvector of $A(G)$ corresponding to $\mu^{*}$.

From Equation (1) we have $2 \lambda_{1}+\lambda_{1}^{*}=n-1$ for regular graph. The following is a generalization of this fact.

Corollary 3.7. Let $\lambda_{1}, \lambda_{2}, \ldots, \lambda_{l}$ and $\lambda_{1}^{*}, \lambda_{2}^{*}, \ldots, \lambda_{l}^{*}$ are all main eigenvalues of $A(G)$ and $A^{*}(G)$, respectively. Then

$$
\sum_{i=1}^{l}\left(2 \lambda_{i}+\lambda_{i}^{*}\right)=n-l .
$$

Proof. Since $\lambda_{1}^{*}, \lambda_{2}^{*}, \ldots, \lambda_{l}^{*}$ are all eigenvalues of matrix $M$ in Theorem 5 , we get

$$
\begin{aligned}
\sum_{i=1}^{l} \lambda_{i}^{*} & =\operatorname{trace}(M)=\sum_{i=1}^{l}\left(\mathbf{e}^{T} \alpha_{i}\right)^{2}-l-\sum_{i=1}^{l} 2 \lambda_{i} \\
& =\sum_{i=1}^{l} n_{i}-l-\sum_{i=1}^{l} 2 \lambda_{i} \\
& =n-l-\sum_{i=1}^{l} 2 \lambda_{i}
\end{aligned}
$$

Hence Corollary follows.

From Theorem 3.5. we know that if $A(G)$ has few main eigenvalues then the main eigenvalues of $A^{*}(G)$ can be obtained easily. The following is an example.

Example 3.8. Let $G=G_{1} \cup G_{2}$ be the union of two regular graphs $G_{1}$ and $G_{2}$ of order $n_{1}$ and $n_{2}$ and degree $r_{1}$ and $r_{2}\left(r_{1} \neq r_{2}\right)$, respectively. It is easy to see that $A(G)$ has exactly two main eigenvalues $r_{1}$ and $r_{2}$ and 
associated orthonormal eigenvector are $\alpha_{1}=\frac{1}{\sqrt{n_{1}}}(\overbrace{1,1, \ldots, 1}^{n_{1}}, 0, \ldots, 0)^{T}$ and $\alpha_{2}=\frac{1}{\sqrt{n_{2}}}(0, \ldots, 0, \overbrace{1,1, \ldots, 1}^{n_{2}})^{T}$. Thus

$$
M=\left[\begin{array}{cc}
n_{1}-1-2 r_{1} & \sqrt{n_{1} n_{2}} \\
\sqrt{n_{1} n_{2}} & n_{2}-1-2 r_{2}
\end{array}\right] .
$$

Hence two main eigenvalues of $A^{*}(G)$ are

$$
\lambda_{1,2}^{*}=\frac{n_{1}+n_{2}-2-2 r_{1}-2 r_{2} \pm \sqrt{\Delta}}{2},
$$

where

$$
\Delta=\left[n_{1}+n_{2}-2-2\left(r_{1}+r_{2}\right)\right]^{2}-4\left[\left(n_{1}-1-2 r_{1}\right)\left(n_{2}-1-2 r_{2}\right)-n_{1} n_{2}\right] .
$$

\title{
REFERENCES
}

[1] D. Cvetković, M. Doob, H. Sachs, Spectra of graphs: Theory and applications, 3rd revised and enlarged edition, J.A. Bart Verglas, Heidelberg, Leipzig, 1995.

[2] D. Cvetković, M. Doob, Developments in the theory of graph spectra, Linear and Multilinear Algebra, 18 (1985),153-181.

[3] D. Cvetković, P. Rowlinson, S. Simić, Eigenspaces of graphs, Cambridge University Press, Cambrige, 1997.

[4] E.M. Hagos, Some results on graph spectra, Linear Algebra and Its Applications, 356 (2002),103-111.

[5] Y.P. Hou and H.Q. Zhou, Trees with exactly two main eigenvalues (Chinese. English summary), J. Nat. Sci. Hunan Norm. Univ., 26 (2005), 1-3.

[6] M. Lepović, A note on graphs with two main eigenvalues, Kragujevac J. Math. 24(2002), 43-53.

[7] M. Lepović, On the Seidel eigenvectors of a graph, Univ. Beograd. Publ. Elektrotehn. Fak., Ser. Mat.,14(2003),4-10.

[8] J.J. Seidel,Strongly regular graphs with $(-1,0,1)$-adjacency matrix have eigenvalue 3, Linear Algebra and Its Applications, 1 (1968),281-298.

[9] P. Rowlinson, The main eigenvalues of a graph: A survey, AADM.1 (2007),445-471.

\author{
HOUQING ZHOU \\ Department of Mathematics \\ SHAOYANG UNIVERSITY \\ HuNAN 422000 \\ P.R. China \\ E-mail address: zhouhq2004@163.com
}

